\title{
Correction to: Eigenfunction Expansions of Ultradifferentiable Functions and Ultradistributions. III. Hilbert Spaces and Universality
}

\section{Aparajita Dasgupta ${ }^{1} \cdot$ Michael Ruzhansky ${ }^{2,3}$ (1)}

Published online: 20 July 2021

○) Springer Science+Business Media, LLC, part of Springer Nature 2021

\section{Correction to: Journal of Fourier Analysis and Applications (2021) 27:15 https://doi.org/10.1007/s00041-021-09817-2}

This article was updated to change the corresponding author to Michael Ruzhansky.

Publisher's Note Springer Nature remains neutral with regard to jurisdictional claims in published maps and institutional affiliations.

The original article can be found online at https://doi.org/10.1007/s00041-021-09817-2.

Michael Ruzhansky

Michael.Ruzhansky@UGent.be

Aparajita Dasgupta

adasgupta@maths.iitd.ac.in

1 Department of Mathematics, Indian Institute of Technology, Delhi, Hauz Khas,

New Delhi 110016, India

2 Department of Mathematics: Analysis, Logic and Discrete Mathematics, Ghent University, Ghent, Belgium

3 School of Mathematical Sciences, Queen Mary University of London, London, UK 